\title{
Variability in Motor and Language Recovery during the Acute Stroke Period
}

\author{
Lauren E. Dunn $^{a} \quad$ Adam B. Schweber ${ }^{b}$ Daniel K. Manson ${ }^{b}$ \\ Andrea Lendaris $^{b} \quad$ Charlotte Herber $^{b}$ Randolph S. Marshall ${ }^{b}$ \\ Ronald M. Lazar ${ }^{b}$ \\ a Baylor College of Medicine, Houston, Tex., and ${ }^{b}$ The Richard and Jenny Levine Cerebral \\ Localization Laboratory, Department of Neurology, Columbia University Medical Center, \\ New York, N.Y., USA
}

Key Words

Stroke recovery $\cdot$ Acute stroke $\cdot$ Motor impairment $\cdot$ Aphasia

\section{Abstract}

Background: Most stroke recovery occurs by 90 days after onset, with proportional recovery models showing an achievement of about $70 \%$ of the maximal remaining recovery. Little is known about recovery during the acute stroke period. Moreover, data are described for groups, not for individuals. In this observational cohort study, we describe for the first time the daily changes of acute stroke patients with motor and/or language deficits over the first week after stroke onset. Methods: Patients were enrolled within 24-72 h after stroke onset with upper extremity hemiparesis, aphasia, or both, and were tested daily until day 7 or discharge with the upper-extremity Fugl-Meyer Assessment of Motor Recovery after Stroke, the Boston Naming Test, and the comprehension domain from the Western Aphasia Battery. Discharge scores, and absolute and proportional changes were examined using t-tests for pairwise comparisons and linear regression to determine relative contributions of initial impairment, lesion volume, and age to recovery over this period. Results: Thirty-four patients were enrolled: 19 had motor deficits alone, 8 had aphasia alone, and 7 had motor and language deficits. In a group analysis, statistically significant changes in absolute scores were found in the motor $(p<0.001)$ and comprehension $(p<0.001)$ domains but not in naming. Day-by-day recovery curves for individual patients displayed wide variation with comparable initial impairment. Proportional recovery calculations revealed that, on average, patients achieved less than $1 / 3$ of their potential recovery by the time of discharge. Multivariate regression showed 
that the amount of variance accounted for by initial severity, age, and lesion volume in this early time period was not significant for motor or language domains. Conclusions: Over the first week after stroke onset, recovery of upper extremity hemiparesis and aphasia were not predictable on the basis of initial impairment, lesion volume, or age. In addition, patients only achieved about $1 / 3$ of their remaining possible recovery based on the anticipated $70 \%$ proportion found at 90 days. These findings suggest that the complex interaction between poststroke structural repair, regeneration, and functional reorganization during the first week after stroke has yet to be elucidated.

(C) 2016 The Author(s)

Published by S. Karger AG, Basel

\section{Introduction}

Previous studies of motor and language function after stroke have suggested that most recovery takes place by 90 days after stroke onset and that initial impairment correlates highly with final outcome over this period [1,2]. Analytic limitations in correlating initial with later function lie in the ceiling effect among those with mild initial impairment and the greater room for improvement among those with severe deficits, making comparisons across different degrees of initial impairment difficult. A more recent model of recovery was introduced to normalize comparisons by determining the ratio of the initial score on an impairment test (e.g. hemiparesis) to the difference between the highest possible (maximal) score and the initial impairment score; this difference was operationally defined as 'potential recovery'. Near-maximum scores on these measures are typically achieved in nonpathological circumstances.

We and others have shown that patients with mild to moderate aphasia or upperextremity weakness consistently regained about $70 \%$ of the maximal possible recovery in the first 3 months after stroke onset [3-5]. Other investigators showed the same proportion of recovery after 4 months [6] and, in a larger cohort, 78\% proportional recovery at 6 months [7]. Within this framework, however, the recovery during early periods after ictus, especially during the first week after stroke, has remained less clear. Physiological studies comparing function within hours after stroke onset and again at 3 days found that clinical improvement correlated with restoration of flow within the ischemic penumbra $[8,9]$. There is otherwise little known about the evolution of deficits during the remaining course of the acute stroke admission. The purpose of this study was to examine motor and language recovery during the first week after stroke, examining the trajectory of recovery at the group level and for individuals. We also wanted to determine the extent to which the traditional predictive factors of initial syndrome severity, lesion volume, and age accounted for recovery in language and motor function during this period. The clinical relevance is the extent to which discharge levels of function are predictable by initial levels of function and risk factors in two common stroke-related impairments and how much of the expected 90-day recovery is actually achieved during this early period.

\section{Methods}

Patients aged $\geq 18$ years admitted to the Columbia University Medical Center (CUMC) Stroke Service were screened for eligibility and enrolled if they demonstrated upper-extremity motor weakness, aphasia, or both (see criteria below). They had to have positive magnetic resonance imaging (MRI) images for infarct (usually diffusion-weighted imaging; DWI), had to be enrolled $\leq 72 \mathrm{~h}$ from stroke onset, and had to be an inpatient for at least 3 daily follow- 
up assessments. Prior stroke was permitted if it occurred in a different vascular territory. Patients were excluded if the clinical examination revealed plegia or global aphasia, impairment of consciousness, prior vision, hearing or movement disability, or other preexisting neurological and psychiatric conditions affecting motor and language function. For the aphasic cohort, only English-speaking patients were included. The CUMC Institutional Review Board approved this study, and informed/surrogate consent was obtained before participation.

Patients with clinical motor deficits received the upper-extremity Fugl-Meyer Assessment of Motor Recovery after Stroke (FM) for testing motor impairment [10]. Aphasic patients were administered the comprehension subtest of the Western Aphasia Battery (WAB) and a 15-item form of the Boston Naming Test (BNT) with 1 of 4 comparable versions randomly given on each day [11-13]. The FM, WAB, and BNT were chosen because of their standardization, well-regarded psychometric properties, high interobserver reliability, as well as because of their extensive use in stroke [14, 15]. Based on published normative data for thresholds of impairment, we only studied patients with an initial FM score $<60$, comprehension score $<180$, and BNT score $<13$ on the initial study evaluation. Administrators were trained by senior investigators with experience administering the tests. We report here the results of the FM (maximum score $=66$ ), comprehension (maximum score $=200$ ), and naming tests (maximum score $=15$ ). Those administering the tests were not aware of the study hypotheses at the time of data collection. Patients were largely tested daily by the same individual up to 7 days after stroke or until discharge, whichever came first; we added 2 days to the allowable length of stay ( 5 days) for uncomplicated ischemic stroke under Medicare in the US. Days after stroke onset were defined as follows: day 1 was on the actual day following stroke onset but no sooner than $24 \mathrm{~h}$ after onset; each day thereafter was defined as the next actual day but no sooner than $24 \mathrm{~h}$ from the previous day. Every attempt was made to evaluate patients at roughly the same time each day, including weekends. Lesion volume, derived from the latest DWI images in nearly every case to capture final infarct volume, was calculated with MIPAV (Medical Image Processing, Analysis, and Visualization) software from the US National Institutes of Health (http://mipav.cit.nih.gov).

Using t-tests, we compared the mean absolute changes between baseline scores and the last day of evaluation for motor function, naming, and comprehension, respectively, and calculated the proportion of achieved recovery, defined as (score discharge $_{\text {- }}$ score $\left._{\text {initial }}\right) /$ ( score $_{\text {maximum }}-$ score $\left._{\text {initial }}\right)[3,4]$, for the whole group. We then examined changes for individual patients. Pearson correlations and multivariate analyses examined the relative contributions of predictive factors of the initial syndrome to the proportion of the potential recovery achieved by patient groups at discharge. Because these measurements were part of a firsttime natural history study for which we had no preexisting hypotheses about the trajectories of recovery, sample sizes could not be calculated.

\section{Results}

From October 2010 to May 2012, 60 patients were screened and 34 patients were enrolled, of whom 19 had motor deficits alone, 8 had aphasia alone, and 7 had motor and language deficits (table 1). Reasons for exclusion after screening included inability to obtain consent; impaired vision, severe hearing loss, and impaired movement unrelated to the index stroke; global aphasia, psychiatric disorder, and insufficient English-speaking background with aphasia. Thirty-three had first-time stroke. Seven patients were treated acutely with intravenous recombinant tissue plasminogen activator, and one had intra-arterial injection of recombinant tissue plasminogen activator followed by clot retraction. Patients were 
Table 1. Demographic characteristics

\begin{tabular}{|c|c|c|c|c|c|c|c|c|}
\hline \multirow[t]{2}{*}{ Subject } & \multirow{2}{*}{$\begin{array}{l}\text { Age, } \\
\text { years }\end{array}$} & \multirow[t]{2}{*}{ Sex } & \multirow[t]{2}{*}{ Handedness } & \multirow{2}{*}{$\begin{array}{l}\text { Side of } \\
\text { stroke }\end{array}$} & \multicolumn{3}{|c|}{ Domain assessed } & \multirow[t]{2}{*}{ Lesion location } \\
\hline & & & & & motor & comprehension & naming & \\
\hline 1 & 57 & $\mathrm{f}$ & $\mathrm{r}$ & 1 & $*$ & $*$ & $*$ & cortical/subcortical \\
\hline 2 & 70 & $\mathrm{~m}$ & $\mathrm{r}$ & l & & & & subcortical \\
\hline 3 & 46 & $\mathrm{f}$ & $r$ & l & & $*$ & $*$ & cortical \\
\hline 4 & 41 & $\mathrm{f}$ & $\mathrm{r}$ & l & & $*$ & $*$ & cortical/subcortical \\
\hline 5 & 76 & $\mathrm{f}$ & $\mathrm{r}$ & l & & & $*$ & cortical \\
\hline 6 & 68 & $\mathrm{~m}$ & $\mathrm{r}$ & l & $*$ & & & cortical/subcortical \\
\hline 7 & 70 & $\mathrm{~m}$ & $\mathrm{r}$ & $\mathrm{r}$ & $*$ & & & subcortical \\
\hline 8 & 99 & $\mathrm{f}$ & $\mathrm{r}$ & $\mathrm{r}$ & $*$ & & & cortical/subcortical \\
\hline 9 & 77 & $\mathrm{f}$ & $\mathrm{r}$ & l & $*$ & $*$ & $*$ & cortical/subcortical \\
\hline 10 & 21 & $\mathrm{f}$ & l & $\mathrm{r}$ & $*$ & $*$ & $*$ & cortical \\
\hline 11 & 80 & $\mathrm{~m}$ & $\mathrm{r}$ & l & $*$ & $*$ & $*$ & cortical \\
\hline 12 & 71 & $\mathrm{~m}$ & $r$ & $r$ & $*$ & & & subcortical \\
\hline 13 & 52 & $\mathrm{~m}$ & $\mathrm{r}$ & l & $*$ & & & subcortical \\
\hline 14 & 80 & $\mathrm{~m}$ & $\mathrm{r}$ & $\mathrm{r}$ & $*$ & & & cortical/subcortical \\
\hline 15 & 61 & $\mathrm{~m}$ & AMB & l & $*$ & & & subcortical \\
\hline 16 & 64 & $\mathrm{~m}$ & $\mathrm{r}$ & l & $*$ & & & subcortical \\
\hline 17 & 80 & $\mathrm{f}$ & $\mathrm{r}$ & l & $*$ & $*$ & $*$ & cortical/subcortical \\
\hline 18 & 56 & $\mathrm{f}$ & $\mathrm{r}$ & l & $*$ & & & subcortical \\
\hline 19 & 65 & $\mathrm{f}$ & $\mathrm{r}$ & $\mathrm{r}$ & $*$ & & & cortical/subcortical \\
\hline 20 & 78 & $\mathrm{f}$ & $\mathrm{r}$ & l & & $*$ & $*$ & cortical \\
\hline 21 & 70 & $\mathrm{f}$ & $\mathrm{r}$ & l & $*$ & & & subcortical \\
\hline 22 & 86 & f & $\mathrm{r}$ & $\mathrm{r}$ & $*$ & & & subcortical \\
\hline 23 & 82 & $\mathrm{~m}$ & $r$ & l & $*$ & $*$ & $*$ & cortical/subcortical \\
\hline 24 & 85 & $\mathrm{f}$ & $\mathrm{r}$ & l & & $*$ & $*$ & cortical/subcortical \\
\hline 25 & 69 & $\mathrm{f}$ & $\mathrm{r}$ & l & $*$ & & & subcortical \\
\hline 26 & 75 & $\mathrm{f}$ & $\mathrm{r}$ & l & $*$ & & & subcortical \\
\hline 27 & 65 & $\mathrm{f}$ & $\mathrm{r}$ & l & & & $*$ & cortical \\
\hline 28 & 77 & $\mathrm{f}$ & $\mathrm{r}$ & l & $*$ & $*$ & $*$ & cortical \\
\hline 29 & 59 & $\mathrm{f}$ & l & $\mathrm{r}$ & $*$ & & & cortical/subcortical \\
\hline 30 & 73 & $\mathrm{f}$ & $\mathrm{r}$ & l & & $*$ & $*$ & cortical \\
\hline 31 & 80 & $\mathrm{f}$ & $\mathrm{r}$ & $\mathrm{r}$ & $*$ & & & subcortical \\
\hline 32 & 71 & $\mathrm{f}$ & $\mathrm{r}$ & $\mathrm{r}$ & $*$ & & & cortical/subcortical \\
\hline 33 & 71 & $\mathrm{~m}$ & $\mathrm{r}$ & l & $*$ & & & cortical \\
\hline 34 & 73 & $\mathrm{f}$ & $\mathrm{r}$ & $\mathrm{r}$ & $*$ & & & subcortical \\
\hline
\end{tabular}

$\mathrm{r}=$ Right; $\mathrm{l}=$ left; $\mathrm{AMB}=$ ambidextrous

otherwise treated with standard thrombotic therapy; to our knowledge, none were enrolled in a stroke treatment trial. There were 23 strokes in the left hemisphere and 11 in the right hemisphere, of which 9 were cortical strokes, 13 subcortical strokes, and 12 with mixed cortical and subcortical events. The mean age was 69 years (range 21-99), and 66\% of patients were women. The mean time of the baseline examination from stroke onset was 2.48 days (SD $=0.67), 94 \%$ of the patients with hemiparesis received some physical or occupational therapy, and $13 \%$ of the patients with aphasia received some speech-language intervention.

The mean FM score at baseline was 25.7 (SD = 19.9), and the mean FM score on the last day of assessment was 36.2 ( $S D=22.13$ ), a difference that was statistically significant ( $\mathrm{p}<$ 0.001 ). Figure 1a shows that the group mean increased every day. Figure $1 \mathrm{~b}$ shows that the initial mean BNT score was 5.37 ( $\mathrm{SD}=1.4$ ), which was unchanged over the hospital stay. The mean for day 7 shows deterioration, although this may be misleading because 3 patients who 
Dunn et al.: Variability in Motor and Language Recovery during the Acute Stroke Period

Fig. 1. Mean scores on the upperextremity (UE) FM test (a), mean scores on the BNT (b), and mean scores on the comprehension subtest of the WAB (c) as a function of days since stroke onset. Error bars denote 1 SD above and below the mean.
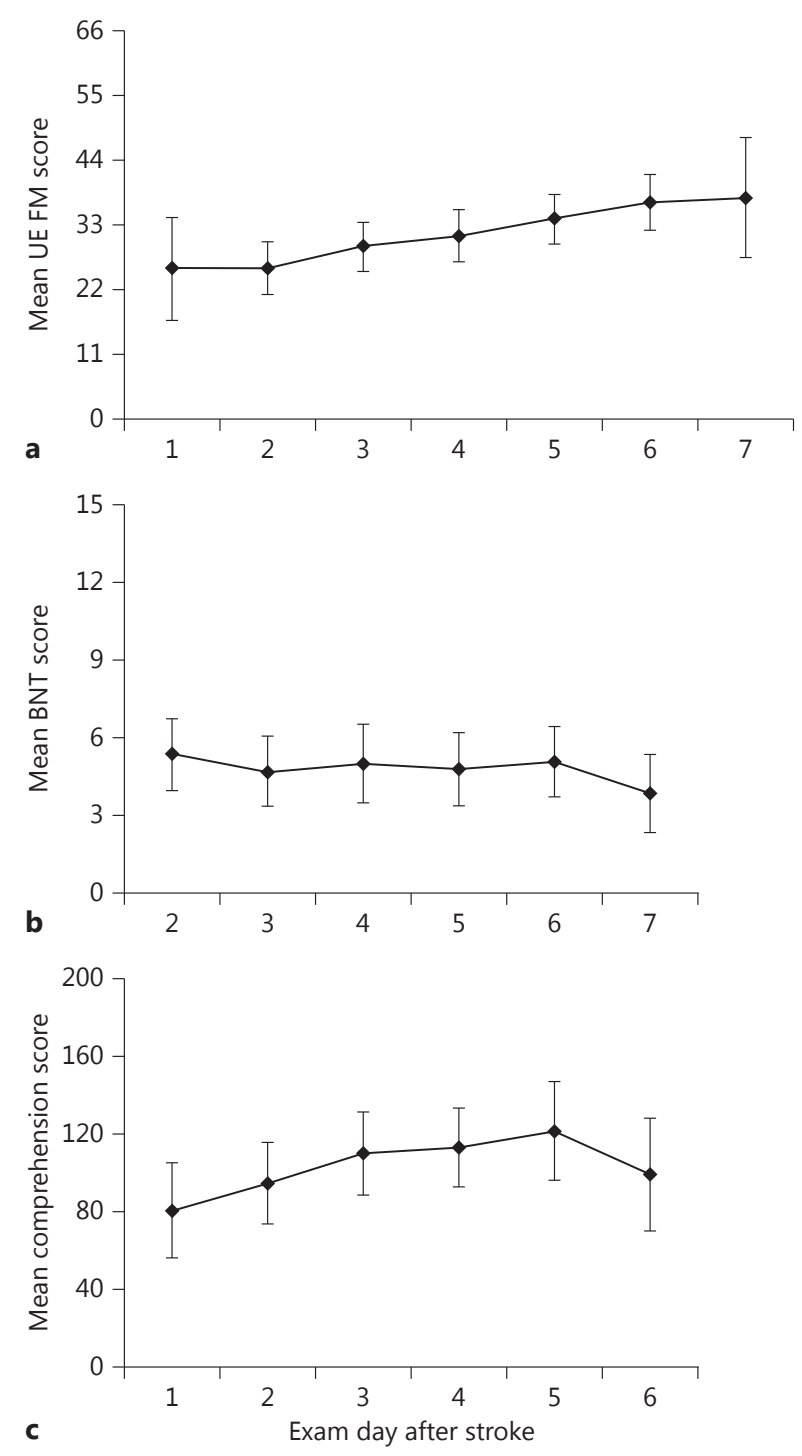

had made good recoveries $(>12 / 15)$ were discharged on day 6 . The mean comprehension scores are depicted in figure 1c. The mean score at baseline was 81.1 (SD =63.8), and by the last day of assessment, the score was 107.6 (SD = 75.60), which was a statistically significant improvement $(\mathrm{p}<0.001)$ even with the day 7 score decrease due to the poorer scores of those remaining until day 7 .

To address proportional recovery and interindividual recovery patterns in motor function, we first plotted day-by-day recovery curves for individual patients, dividing them by severe $(F M=0-22)$, moderate $(F M=23-44)$, or mild $(F M=45-60)$ deficit at baseline (fig. 2a, b and c, respectively).

Note that only 6 patients were seen on poststroke day 1 , and 6 patients on day 7 . The data show that among the severely affected group, some patients had substantial improvement (e.g. FM scores from 13 at baseline to 47 at discharge), while some patients made no recovery 


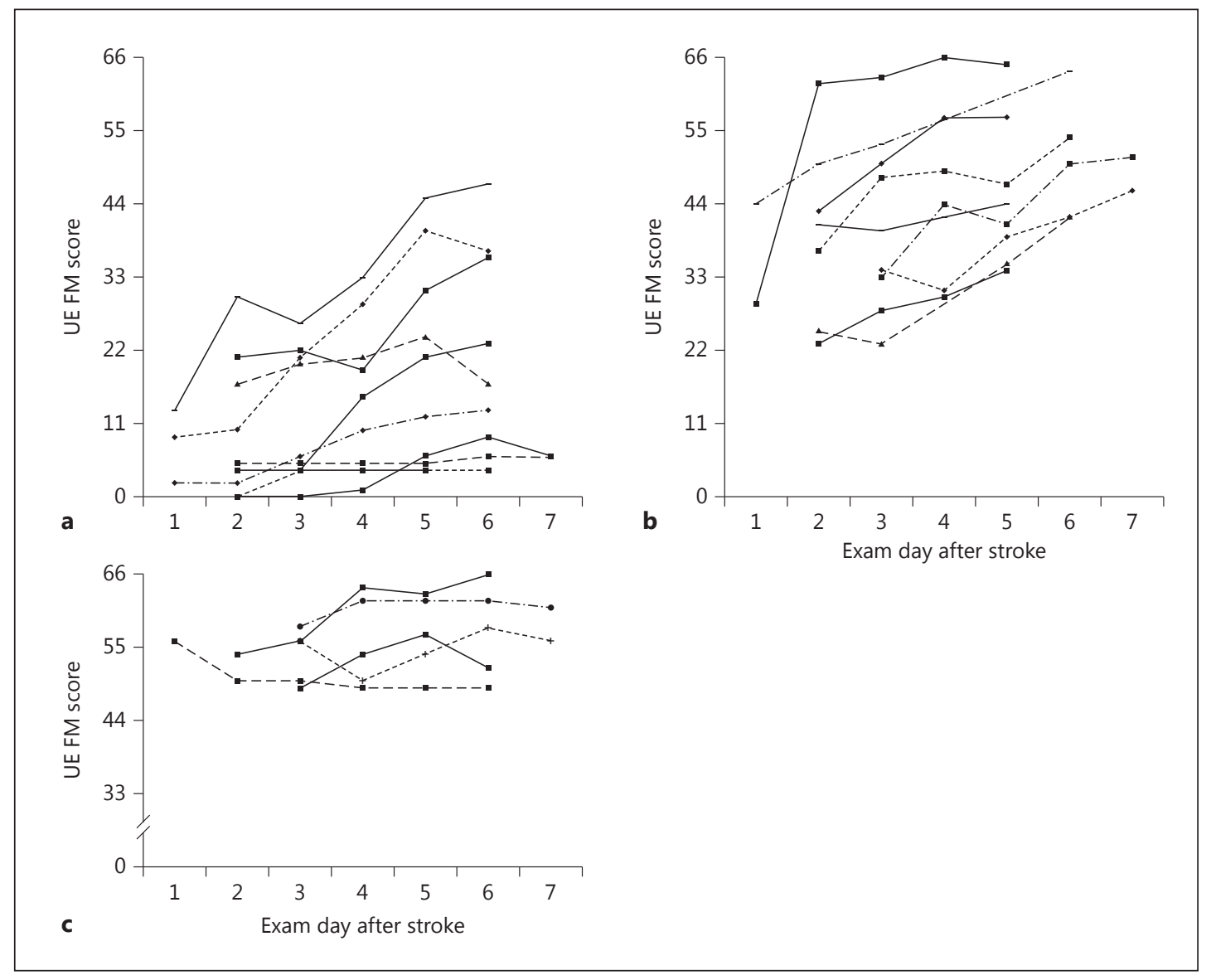

Fig. 2. Daily scores for individual patients with initial FM scores of 0-22 (a), 23-44 (b), and 45-60 (c), roughly comparable to baseline function in the severe, moderate, and mild ranges, respectively. UE = Upper extremity.

at all. In contrast, every patient in the moderate group improved to some degree. In the mild group, 2 patients made virtually complete recoveries, while 3 appeared to make little recovery. The proportional recovery was 0.17 for the individuals with severe initial impairment, 0.53 for those with moderate initial impairment, and 0.17 for those with mild initial impairment. Using 60/66 as a threshold recovery, among all patients with hemiparesis, $4 / 26$ achieved an absolute level of function that would have precluded their eligibility for this study at baseline, one each from the mild, moderate, and severe hemiparetic groups, respectively.

Figure 3a displays the daily recovery on the BNT and comprehension scale from the WAB (fig. 3b). Five patients scored $0 / 15$ at baseline on the BNT and never improved during the course of their admission. Of the remaining group, there was a patient who scored 11/15 on naming on day 1 and fully recovered by day 3 . Conversely, another patient scored 10/15 on day 2 (baseline) and improved only to $11 / 15$ by day 6 .

The recovery patterns for comprehension were also variable and not predictable based on baseline severity. Those with baseline scores $<80$ made little to no improvement, yet 3 patients with scores $>150$ made no recovery at all. Only 1 patient achieved a perfect $200 / 200$ at discharge.

Lesion volume was not associated with initial syndrome severity for naming, for FM ( $\mathrm{r}=$ $-0.19, p=0.350)$, or for comprehension $(r=-0.212, p=0.532)$. We excluded the lesion size 


\section{Cerebrovascular \\ Diseases}

Fig. 3. Daily scores for individual patients with initial comprehension (a) and naming (b) deficits. There were 5 individuals with baseline scores of 0 who demonstrated no recovery over the acute stroke period.

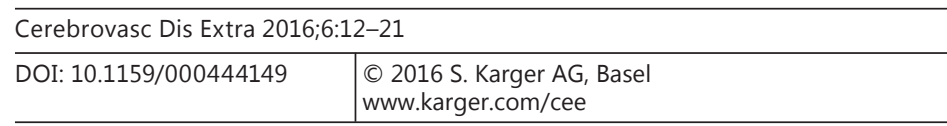

Dunn et al.: Variability in Motor and Language Recovery during the Acute Stroke Period

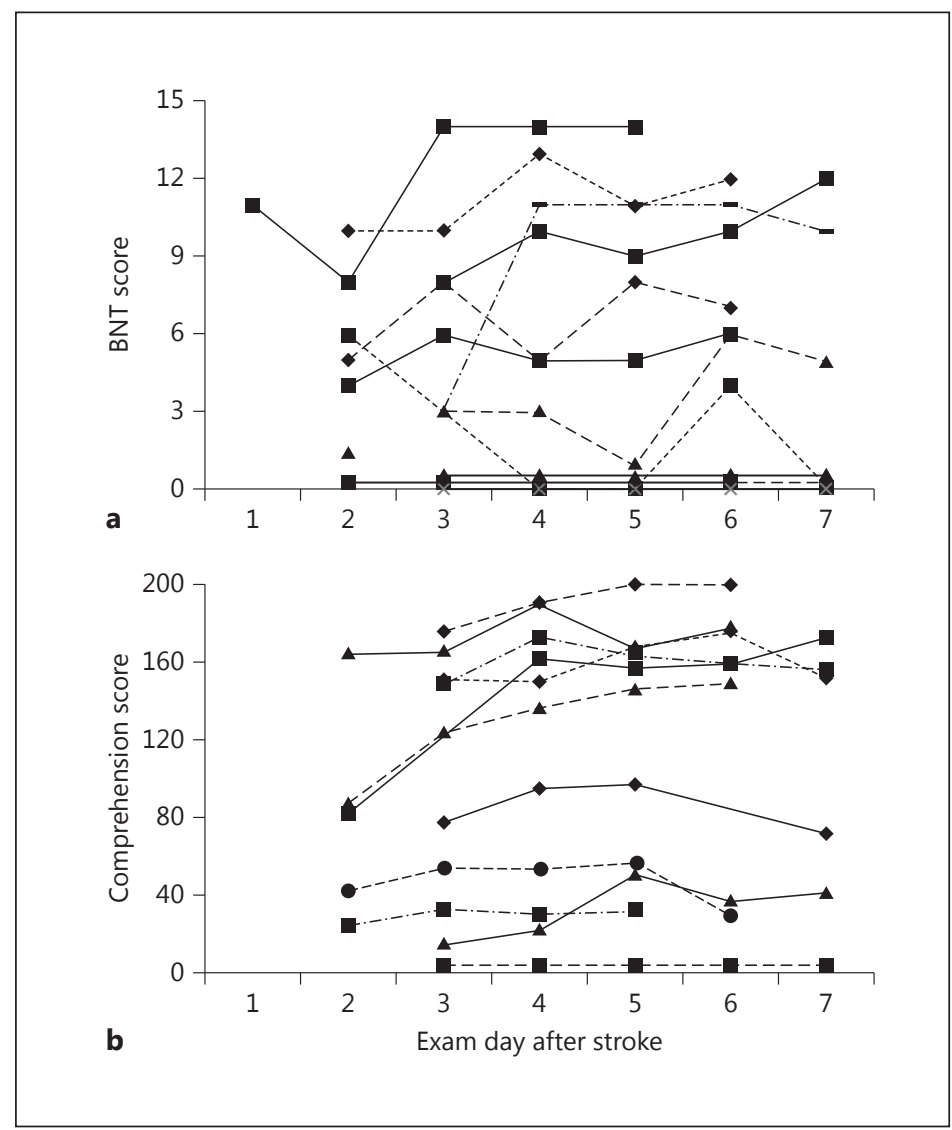

for patient $29\left(640 \mathrm{~mm}^{3}\right)$ because it was $>20 \mathrm{SD}$ greater than the next largest volume. As a group, naming deficits were also not correlated with lesion volume. In a subgroup analysis among those with naming deficits, the $5 / 11$ patients who scored $0 / 15$ at baseline and never improved had an average lesion volume of $82.0 \mathrm{~mm}^{3}$, whereas the remaining 6 patients, with baseline naming scores ranging from 3 to 11 , had lesion volumes of $24.6 \mathrm{~mm}^{3}$, a difference that was statistically significant $(p=0.007)$. The correlation between lesion volume and proportion of FM recovery was not significant ( $r=-0.30, p=0.14$ ), as was the case for comprehension $(\mathrm{r}=-0.25, \mathrm{p}=0.47)$. Multivariate regression showed that the amount of variance accounted for $\left(\mathrm{r}^{2}\right)$ by the contributions of initial severity, age, and lesion volume to the proportion of recovery during the acute stroke period for FM, BNT, and WAB were -0.02 , 0.25 , and 0.05 , respectively, none of which was statistically significant.

\section{Discussion}

Among hemiparetic and aphasic patients studied daily during the first 7 days after stroke onset, initial impairment, lesion volume, and age were not predictive of the absolute amount or the proportion of their recovery during this period for motor or language deficits. Although those with motor and comprehension deficits improved as groups over the admission period, there was sufficient variation among individuals with comparable levels of initial impairment to preclude a prediction of discharge levels of function. Moreover, few patients achieved what is conventionally described as a complete or near-complete recovery during this period. 

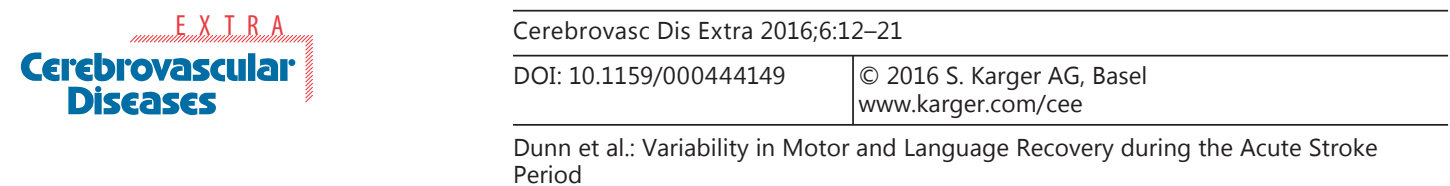

In general, it has been assumed that recovery follows a roughly positive trajectory during the first week after stroke onset [1], but our data suggest that the slope of this improvement may be lower and more variable than previously thought. Traditional predictors such as initial syndrome severity, lesion volume, and age did not correlate either with change over time or final performance. Prior studies in stroke recovery have reported the importance of these predictors 90 days after stroke, but their relevance during the first week after stroke had not been explored. Our daily testing with finer-grained instruments during this period made the observation of this interindividual variability possible. Indeed, this study demonstrates the feasibility and importance of daily quantitative testing of motor and language function in the days after stroke onset with a degree of resolution not utilized in the past.

The early variability could be attributed to a number of physiological and clinical factors that coexist during the acute stroke period, such as inflammation [16, 17], hemorrhagic conversion [18], perfusion [8], and pharmacological [19] and psychological factors [20]. Recent views of stroke recovery have cited tissue injury and dysfunction by these and other factors in the first few days after stroke, followed by early structural and metabolic repair, and the upregulation of plasticity mechanisms [21]. However, the intertwined temporal course of neural destructive and neural repair systems in the early period after stroke has yet to be defined. For example, it has been shown that persistent hypoperfusion can last up to several days after stroke onset [22], while functional MRI evidence of new contralesional activity has been found within $24 \mathrm{~h}$ after stroke onset [23]. The variability and minimal improvement found in our data suggest that the dynamic interaction among injury, repair, regeneration, and reorganization probably lasts longer and is less serial than previously thought. These complexities have implications for the timing of poststroke restorative therapies.

Generally speaking, there is consensus that most spontaneous recovery of motor and language function takes place in the first 90 days after stroke onset [1,24, 25], with perhaps some potential for later recovery with the use of pharmacological and stimulation-based therapies [26-28]. In this study, we wished to address the question of the degree to which restoration of function can be expected by the end of the first week, which in part depends on how 'recovery' is defined. A single outcome at one time point reflects a specific level of function or competency on a task and may serve as a therapeutic target. It is not clear, however, how to interpret an outcome that is not near perfect.

We would argue that functional stroke recovery is a biological process in which changes in behavior are driven by repair and adaptation in the brain physiology that occurs in a temporal fashion. An older approach to examining change has been to find the difference between initial and final performance [1]. The analytic limitations in this strategy lie in the ceiling effect among those with mild initial impairment and the greater room for improvement among those with severe deficits, making comparisons difficult. To address this analytic challenge, the notion of proportional recovery emerged by normalizing change across the severity spectrum and determining whether comparable changes over time could be predicted based on initial impairment. We previously found that individuals with mild to moderate initial deficits in motor and language function tended to recover $70 \%$ of their potential recovery by 90 days $[3,4]$. More recently, it was found that there was little additional change from $\sim 70 \%$ from 90 to 120 days [5]. In contrast, those with severe initial motor deficits (upper-extremity FM score $\leq 20$ ) have been less predictable by this model alone. In the present study, the average proportion of recovery at the end of the first week after stroke was less than $1 / 3$ of the potential recovery, with less among those with the mildest and most severe initial language and motor deficits. Based on the expectation of 70\% proportional recovery, at least in the groups who were not severe at onset, a significant amount of recovery had yet to take place, perhaps attributable to the unexpected variation across patients. The clinical implication is 
that the absolute level of function at discharge, the absolute change over this first week, and the proportion of change in this early period provide little predictive power for the expected level of function by the end of 3 months.

There are several limitations of this study. First, we enrolled 34 patients; a larger cohort might have resulted in the establishment of significant differences. Results of analyses, however, did not approach trends in cases of nonsignificance. Despite the relatively small cohort, however, we were able to enroll patients with a wide range of initial impairment, representing the typical spectrum of aphasic and hemiparetic deficits seen in a large stroke service. If anything, we tended to enroll those with moderate to severe deficits. Thus, our ability to make strong inferences regarding recovery patterns among patients with minor deficits was limited. In addition, our enrollment precluded a meaningful comparison of recovery trajectories within individuals who had both aphasia and hemiparesis. Another limitation is that we did not follow these patients to 90 days to confirm empirically the expectation of $70 \%$ proportional recovery. Our inferences, however, were based on the prior proportional analysis of $>250$ patients with hemiparesis and aphasia at stroke onset. We also recognize that there can be dynamic changes in DWI volume (decreasing in size because of reperfusion or expanding because the penumbra can progress to infarction) after we obtained our images at 24-72 $\mathrm{h}$ after onset. The strengths of this study include data derived from the prospective, daily assessments of acute stroke patients with well-established quantitative measures of motor and language function, which to date have not been reported.

In conclusion, our cohort study of stroke patients in the first week after onset showed that we do not as yet have good predictors of motor and language recovery during the course of the acute stroke admission. On average, there was little recovery during this period. With, on average, less than $1 / 3$ of the expected proportional recovery occurring during this early period, most improvement will occur after discharge, some time between 1 and 12 weeks after stroke onset, in trajectories that will be further delineated in future research.

\section{Acknowledgements}

This research was funded by NIH grant 1R01 HD43249-01A2 (R.M.L.), 1-P50NS049060-01 (R.S.M.), Doris Duke Charitable Foundation (L.E.D.), T35 AG044303 (D.K.M.), Tananbaum Family Foundation, and the Richard and Jenny Levine Foundation.

\section{Disclosure Statement}

The authors declare that they have no conflicts of interest.

\section{References}

1 Duncan PW, Goldstein LB, Matchar D, Divine GW, Feussner J: Measurement of motor recovery after stroke. Outcome assessment and sample size requirements. Stroke 1992;23:1084-1089.

-2 Demeurisse G, Demol O, Derouck M, de Beuckelaer R, Coekaerts MJ, Capon A: Quantitative study of the rate of recovery from aphasia due to ischemic stroke. Stroke 1980;11:455-458.

-3 Prabhakaran S, Zarahn E, Riley C, Speizer A, Chong JY, Lazar RM, et al: Inter-individual variability in the capacity for motor recovery after ischemic stroke. Neurorehabil Neural Repair 2008;22:64-71.

-4 Lazar RM, Minzer B, Antoniello D, Festa JR, Krakauer JW, Marshall RS: Improvement in aphasia scores after stroke is well predicted by initial severity. Stroke 2010;41:1485-1488.

-5 Winters C, van Wegen EE, Daffertshofer A, Kwakkel G: Generalizability of the proportional recovery model for the upper extremity after an ischemic stroke. Neurorehabil Neural Repair 2015;29:614-622. 
6 Feng W, Wang J, Chhatbar PY, Doughty C, Landsittel D, Lioutas VA, et al: Corticospinal tract lesion load: an imaging biomarker for stroke motor outcomes. Ann Neurol 2015;78:860-870.

7 Byblow WD, Stinear CM, Barber PA, Petoe MA, Ackerley SJ: Proportional recovery after stroke depends on corticomotor integrity. Ann Neurol 2015;78:848-859.

8 Hillis AE, Barker PB, Beauchamp NJ, Gordon B, Wityk RJ: MR perfusion imaging reveals regions of hypoperfusion associated with aphasia and neglect. Neurology 2000;55:782-788.

-9 Croquelois A, Wintermark M, Reichhart M, Meuli R, Bogousslavsky J: Aphasia in hyperacute stroke: language follows brain penumbra dynamics. Ann Neurol 2003;54:321-329.

10 Gladstone DJ, Danells CJ, Black SE: The Fugl-Meyer Assessment of Motor Recovery after Stroke: a critical review of its measurement properties. Neurorehabil Neural Repair 2002;16:232-240.

11 Shewan CM, Kertesz A: Reliability and validity characteristics of the Western Aphasia Battery (WAB). J Speech Hear Disord 1980;45:308-324.

12 Fastenau PS, Denburg NL, Mauer BA: Parallel short forms for the Boston Naming Test: psychometric properties and norms for older adults. J Clin Exp Neuropsychol 1998;20:828-834.

13 Mack WJ, Freed DM, Williams BW, Henderson VW: Boston Naming Test: shortened versions for use in Alzheimer's disease. J Gerontol 1992;47:P154-P158.

14 Platz T, Pinkowski C, van Wijck F, Kim IH, di Bella P, Johnson G: Reliability and validity of arm function assessment with standardized guidelines for the Fugl-Meyer Test, Action Research Arm Test and Box and Block Test: a multicentre study. Clin Rehabil 2005;19:404-411.

15 Pedersen PM, Vinter K, Olsen TS: Aphasia after stroke: type, severity and prognosis. The Copenhagen Aphasia Study. Cerebrovasc Dis 2004;17:35-43.

16 del Zoppo GJ: Stroke and neurovascular protection. N Engl J Med 2006;354:553-555.

17 Zhou J, Li J, Rosenbaum DM, Barone FC: Thrombopoietin protects the brain and improves sensorimotor functions: reduction of stroke-induced MMP-9 upregulation and blood-brain barrier injury. J Cereb Blood Flow Metab 2011;31:924-933.

18 Jickling GC, Liu D, Stamova B, Ander BP, Zhan X, Lu A, et al: Hemorrhagic transformation after ischemic stroke in animals and humans. J Cereb Blood Flow Metab 2014;34:185-199.

19 Lazar RM, Fitzsimmons BF, Marshall RS, Berman MF, Bustillo MA, Young WL, et al: Reemergence of stroke deficits with midazolam challenge. Stroke 2002;33:283-285.

20 Hackett ML, Anderson CS, House AO: Management of depression after stroke: a systematic review of pharmacological therapies. Stroke 2005;36:1098-1103.

21 Carmichael ST: Cellular and molecular mechanisms of neural repair after stroke: making waves. Ann Neurol 2006;59:735-742.

22 Motta M, Ramadan A, Hillis AE, Gottesman RF, Leigh R: Diffusion-perfusion mismatch: an opportunity for improvement in cortical function. Front Neurol 2014;5:280.

23 Marshall RS, Perera GM, Lazar RM, Krakauer JW, Constantine RC, DeLaPaz RL: Evolution of cortical activation during recovery from corticospinal tract infarction. Stroke 2000;31:656-661.

24 Kwakkel G, Kollen BJ: Predicting activities after stroke: what is clinically relevant? Int J Stroke 2013;8:25-32.

25 Stinear CM, Byblow WD: Predicting and accelerating motor recovery after stroke. Curr Opin Neurol 2014;27: 624-630.

26 Berthier ML, Pulvermuller F: Neuroscience insights improve neurorehabilitation of poststroke aphasia. Nat Rev Neurol 2011;7:86-97.

-27 Saur D, Hartwigsen G: Neurobiology of language recovery after stroke: lessons from neuroimaging studies. Arch Phys Med Rehabil 2012;93:S15-S25.

28 Wolf SL, Winstein CJ, Miller JP, Taub E, Uswatte G, Morris D, et al: Effect of constraint-induced movement therapy on upper extremity function 3-9 months after stroke: the EXCITE randomized clinical trial. JAMA 2006;296:2095-2104. 